\title{
A NEW ELECTRONIC VERIFICATION METHOD FOR VECTOR NETWORK ANALYZERS
}

\author{
R. Ginley, D. Williams, D. LeGolvan \\ National Institute of Standards and Technology \\ Boulder, CO, USA
}

\begin{abstract}
$\underline{\text { Abstract }}$
The National Institute of Standards and Technology (NIST) has recently introduced a new method for the verification of Vector Network Analyzers (VNAs). The technique is based on the new electronic calibration units that are available from several manufacturers. Using data obtained from different states of the electronic calibration unit, the user can compare their measurements against those of NIST with NIST's uncertainty identified. Additionally, the users can compare the calibration based on their data against NIST's calibration based on the same electronic calibration unit. The resultant is a single figure of merit that characterizes the accuracy of the entire measurement process. In this paper we will describe the new technique and results from trial runs that have been conducted.
\end{abstract}

\section{$\underline{\text { Introduction }}$}

Modern Vector Network Analyzers (VNAs) are capable of producing very accurate microwave measurements. They can do this while generating very large amounts of data. Unfortunately they are also capable of producing very poor results. The accuracy of VNA measurements comes almost entirely from the calibration process, also known as "error reduction", because the instruments, while highly repeatable, are far from ideal. The calibrations are also error prone, and verification techniques are important as they allow the user to check the validity of a "calibration" before processing other measurements.

In the past, VNA verification methods have incorporated measurements of multiple physical standards [1]. This involves many connections to several different devices, with resulting wear on the device connectors, potential bad connections, and significant time invested in the measurements.

NIST has developed a new verification technique that replaces the old mechanical devices method. The technique utilizes the new electronic calibration units now available. The use of the electronic calibration units allows one to do everything that could be done with the physical verification devices and does it with a single connection instead of the multiple connections and greatly reduces the time required for the process. Additionally, the new technique provides a means for putting an error bound on the measurements from the test system based on a comparison to a reference calibration.

\section{$\underline{\text { Technique Description }}$}

The new technique uses measurements from the VNAs at NIST and those made on a user's VNA [2]. After being characterized by NIST, an electronic calibration unit is sent to the customer. The customer will load the NIST software onto a controller computer, connect the computer to the VNA, via USB, IEEE488, internet LAN, etc., and then attach the electronic calibration unit to the controller - not to the VNA. This allows the NIST software to control the electronic calibration unit, not the VNA, so any manufacturer of electronic calibration unit can be used on any VNA. The software will direct the customer to calibrate their VNA in whatever manner they see fit for the test (usually the same technique/standards they will use for making measurements). After the calibration, the software will direct the customer to attach the electronic calibration unit to the VNA and will then proceed to automatically take measurements of the different states of the electronic calibration unit.

With the data obtained, two virtual calibrations are formed based on the customer's data and NIST's data for the states of the electronic calibration unit. These calibrations are then compared using processes developed by Williams, Marks, and Davidson [3-5]. The comparison in this case gives a worst-case difference for measurements of any complex two- or one-port device relative to the NIST calibration (see Fig. 1). Additionally, the customer's data for the measurement of each state of the electronic calibration unit can be compared to NIST's data for that state with the NIST uncertainty identified (see Fig. 2). This technique allows a customer to verify their entire measurement process from the initial error correction to the final measurement.

\section{$\underline{\text { Results }}$}

Figure 1 shows a plot of the worst-case deviation for the calibration comparison portion of the technique.

* U.S. Government work; not protected by U.S. copyright 
It shows a bound for the difference in measurements from the customer-calibrated VNA to those of the traceable NIST calibration. The magnitude of the results can be used as a guide to the validity of the customer's calibration.

Figure 2 shows the difference between the customer's results and those of NIST for a particular state of the electronic calibration unit with the NIST uncertainties identified. This can be used in the same manner as results from the physical standards. Additional plots comparing the measurements are also available.

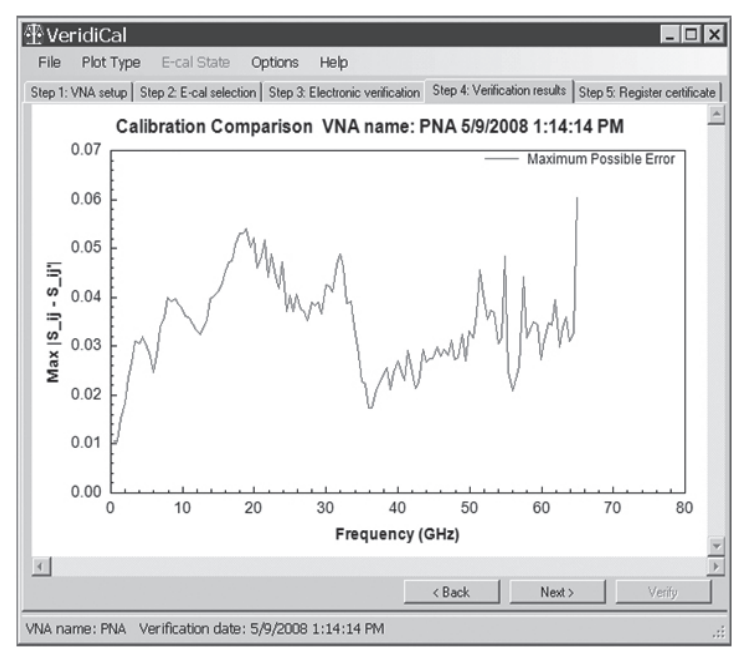

Figure 1. The calibration comparison metric is shown. It provides a bound on the differences of measurements from a customer's calibrated VNA to those from a NIST VNA.

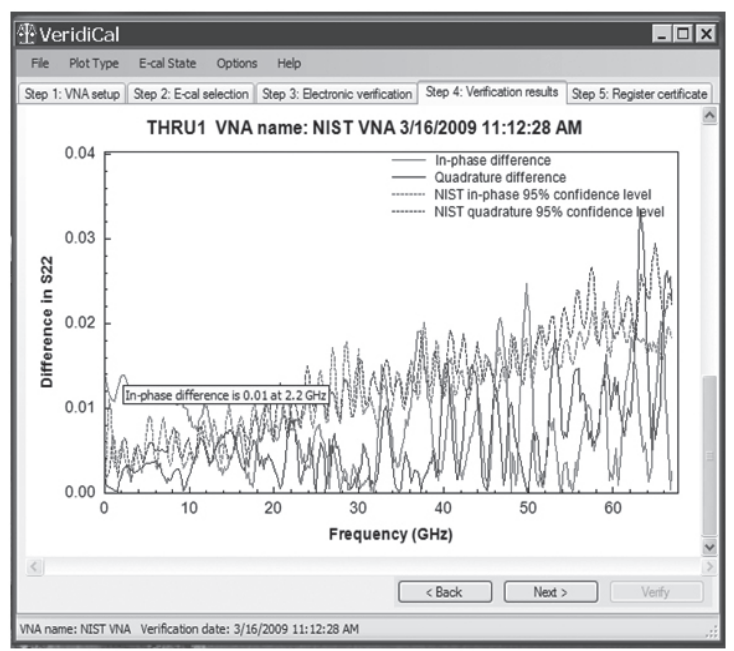

Figure 2. Plot showing the differences between a customer's and NIST's measurement results for a particular state of an electronic calibration unit.

\section{$\underline{\text { Conclusion }}$}

A new VNA verification technique has been developed at NIST. This technique uses electronic calibration units instead of the multiple physical artifacts that were used in older techniques. The new technique gives all of the verification results of the physical devices approach, and also puts a bound on the difference of measurements between a customer calibration and a NIST calibration. At present, pass/fail criteria have not been developed for this verification approach. However, it should be possible to develop pass/fail criteria based on the NIST uncertainty analysis and further studies of the stability of the electronic calibration units. The new technique has a large advantage over the physical artifact technique, as it limits the number of connections made to the verification device and thus reduces wear and reduces the time necessary for the entire process.

\section{$\underline{\text { References }}$}

[1] R. A. Ginley, "Confidence in VNA Measurements," IEEE Microwave Magazine, vol. 8, no. 4, pp. 54-58, Aug. 2007.

[2] D. Williams, A. Lewandowski, D. LeGolvan, R. Ginley, "Electronic Vector-NetworkAnalyzer Verification," IEEE Microwave Magazine, vol. 10, no. 6, pp.118-123, Oct. 2009

[3] D. F. Williams, R. B. Marks, and A. Davidson, "Comparison of On-Wafer Calibrations," Automatic RF Techniques Group Conference Digest, vol. 38, pp. 68-81, Dec. 1991.

[4] D. F. Williams, C. M. Wang, and U. Arz, "An Optimal Vector-Network-Analyzer Calibration Algorithm," IEEE Trans. Microwave Theory Tech., vol. 51, no. 12, pp. 2391-2401, Dec. 2003.

[5] D. F. Williams, C. M. Wang, and U. Arz, "An Optimal Multiline TRL Calibration Algorithm," IEEE MTT-S Int. Microwave Symp. Dig., vol. 3, pp. 1819-1822, June 2003. 